\title{
Inertia, Optimism and Beauty
}

\author{
Patrick Hawley
}

March 1, 2010

\begin{abstract}
The best arguments for the $1 / 3$ answer to the Sleeping Beauty problem all require that when Beauty awakes on Monday she should be uncertain what day it is. I argue that this claim should be rejected, thereby clearing the way to accept the $1 / 2$ solution.
\end{abstract}

\section{Inertia and Sleeping Beauty}

If on Monday you confidently and rationally believe that Slippery Slim is guilty, and from Monday to Tuesday you gain no new relevant evidence, then, quite plausibly, on Tuesday you should remain equally confident that Slippery Slim is guilty. Lots of evidence might be relevant: that Slim has an impeccable alibi, that the informant who fingered Slim is unreliable, that Mournful Mel has confessed to the crime, that your biases have clouded your judgment, and so on. But if you learn nothing at all relevant to your confident belief that Slim is guilty, then surely you should remain confident that Slim is guilty.

Perhaps that was too quick. Suppose you lose some relevant evidence- say you forget why you became convinced that Slim is guilty - then perhaps you should become less confident that he is guilty. Or maybe your confidence should change if your 
cognitive faculties are damaged or disrupted. Still, the following principle is intuitively plausible:

INERTIA If you should have degree of belief $d$ that $p$ at time $t_{1}$, and between $t_{1}$ and a later time $t_{2}$, your cognitive faculties remain in order, and you neither gain nor lose relevant evidence, then you should also have degree of belief $d$ that $p$ at time $t_{2}$.

INERTIA comes into play in the much discussed Sleeping Beauty problem.

On Sunday, Sleeping Beauty[10] is informed that after she goes to sleep that evening a fair coin will be tossed. If the coin lands heads, she will be awakened for a moment on Monday, put back to sleep, and then not awakened until Wednesday morning. If the coin lands tails, Beauty will be awakened for a moment on Monday, and put back to sleep. While sleeping, her memory of the Monday awakening will be erased. Then, on Tuesday, she will be awakened for a moment, put back to sleep and not awakened until Wednesday morning. Whether the coin lands heads or tails, on Wednesday morning she will be awakened and immediately informed that the experiment is over.

When Beauty wakes on Monday, what should be her degree of belief that the coin lands heads? Thirders say $1 / 3$. Halfers say $1 / 2$. Puzzlingly, there are strong arguments on both sides.

A compelling argument for the halfer's view relies on INERTIA. When Beauty awakens on Monday she learns nothing relevant to the coin toss. She does learn that she is awake, but surely that news is not relevant to the coin toss. As Roger White puts it, "[s] he knew all along that she was to be awakened briefly during the experiment at some time. So it is no news to her when she finds herself awake at an unknown time. When awakened she may learn something that she would express as 'I am awake now.' But it is difficult at best to see what bearing this could have for her on whether the coin landed heads." [34] Moreover, Beauty does not forget anything between Sunday and Monday, and she does not lose evidence in any other way. Nor does she suffer any disruption in her cognitive faculties. Strange happenings do occur on Monday night, if the coin lands tails. But from Sunday to Monday morning, nothing of the sort occurs. 
Now, since Beauty knew that the coin was fair, on Sunday she should have degree of belief $1 / 2$ that the coin lands heads. (This is an application of David Lewis' Principal Principle connecting chance to credence.) So, by INERTIA, when she wakes up she should continue to have degree of belief $1 / 2$ that the coin lands heads.

Thirders[1][8][10][14][15][26][29][32] agree with halfers that on Sunday Beauty should have degree of belief $1 / 2$ that the coin lands heads. However, thirders think that Beauty's degree of belief should change to $1 / 3$ when she wakes up on Monday.

The thirder view thus appears incompatible with INERTIA: Beauty's degree of belief that the coin lands heads should change from Sunday to Monday, although she suffers no cognitive disruptions, and she neither gains nor loses relevant evidence.

Despite appearances, few thirders reject INERTiA. Many thirders think Beauty gains some relevant evidence upon awakening on Monday. ${ }^{1}$ Some thirders instead (or additionally) hold that, despite appearances, Beauty loses relevant evidence.

Indeed, a thirder can use INERTIA to argue that Beauty gains relevant evidence: since she should change her degree of belief, she does not forget anything (or lose relevant evidence in some other way) and she suffers no cognitive disruption, it follows, given INERTIA, that she gains relevant evidence. But it is one thing to argue that Beauty gains relevant evidence, and quite another to explain how that evidence is relevant. With a satisfactory explanation how that evidence is relevant, thirders might blunt the force of the halfer reasoning, thereby resolving the puzzle.

However, no explanation how that evidence is relevant has seen wide acceptance; the Sleeping Beauty problem still rankles. Indeed, as I will discuss, the best developed attempt at such an explanation fails. Moreover, as I will explain later, there isn't good reason to think that Beauty loses relevant evidence either.

My goal is to resolve the Sleeping Beauty problem in favor of the halfer view. As I will explain, the best thirder arguments require a common assumption. I will argue

\footnotetext{
${ }^{1}$ e.g. Horgan[15], Weintraub[32], and Stalnaker[29]
} 
that we have better reason to reject this assumption than we have to conclude that Beauty gains relevant evidence or that she loses relevant evidence (or that INERTIA is false). Once the assumption is rejected, these thirder arguments can be set aside, and the path is open to be a halfer. But before identifying the common assumption, it will be useful to clarify some background issues, and then ask a non-standard question about Sleeping Beauty's situation.

\section{Background}

I assume that all agree that when Beauty wakes up on Monday, she should divide her credence among at least some of the following propositions:

$H_{M O N}$ : The coin lands heads and it is now Monday

$T_{M O N}$ : The coin lands tails and it is now Monday

$T_{T U E}$ : The coin lands tails and it is now Tuesday

Let $\mathrm{P}()$ represent the credences Beauty should have when she wakes up on Monday, HEADS be the proposition that the coin lands heads, TAILS be the proposition that the coin lands tails, MON be the proposition that it is now Monday, and TUE be the proposition that it is now Tuesday. I assume that all agree that:

$$
\begin{aligned}
& P\left(H_{M O N}\right)+P\left(T_{M O N}\right)+P\left(T_{T U E}\right)=1 \\
& P(H E A D S)=P\left(H_{M O N}\right) \\
& P(T A I L S)=P\left(T_{M O N}\right)+P\left(T_{T U E}\right) \\
& P(M O N)=P\left(H_{M O N}\right)+P\left(T_{M O N}\right) \\
& P(T U E)=P\left(T_{T U E}\right) .
\end{aligned}
$$




\section{An optimistic answer to a different question}

Thirders and halfers disagree about Beauty's degree of belief, on Monday morning, that the coin lands heads; thirders and halfers disagree about $P(H E A D S)$. Is it $1 / 3$ as thirders claim? Or $1 / 2$ as halfers say? But consider a second question: when Beauty wakes up on Monday what should be her degree of belief that it is Monday? That is, what is $P(M O N)$ ? Little attention has been paid to this second question.

It is an unquestioned assumption in the literature on the Sleeping Beauty problem that when Beauty awakes on Monday she should be uncertain about what day it is, that is that $0<P(M O N)<1$. It is not surprising that this assumption is unquestioned. For imagine yourself in Beauty's shoes as you awake on Monday morning. "I have no idea whether it is Monday or Tuesday!" you might think to yourself. It might seem to you that you have no evidence which indicates that it is now Monday as opposed to Tuesday. It seems intuitively obvious that you should have at least some uncertainty about the day. However, we should not agree that $0<P(M O N)<1$ merely on the basis of brute intuition. It is not clear that our intuitions about such a bizarre situation are reliable. We need to rely on reasoning here, not brute intuition.

Let us question the unquestioned assumption. Suppose Beauty adopts the following optimistic policy: believe to degree 1 that it is Monday whenever awakened during the experiment. If Beauty is an optimist, whether the coin lands heads or tails, when awakened on Monday she will have full credence in the truth that it is Monday. If the coin lands heads, she will never be wrong about the day during the experiment. If the coin lands tails, on Tuesday she will also believe to degree 1 that it is Monday, and thus have full credence in a falsehood.

To explore the plausibility of the optimistic policy, recall her unusual situation: if there are two awakenings, her memory of the Monday awakening is erased on Monday evening. A straightforward way to understand the memory erasure is this: on Monday 
evening Beauty's belief state is reset to what it was just before she fell asleep on Sunday evening. ${ }^{2}$ The result of the erasure, I take it, is that for any proposition $\mathrm{p}$, if, on Monday, Beauty has degree belief $d$ that $\mathrm{p}$, then on Tuesday, Beauty (if she is awakened) has the same degree of belief $d$ that p. Thus, if the coin lands tails, then both on Monday and on Tuesday Beauty has, for example, the same degree of belief that it is Monday, and the same degree of belief that the coin lands heads. In short, her degrees of belief are yoked together between Monday and Tuesday, if she awakens on Tuesday. ${ }^{3,4}$

If the coin lands tails, the yoking of her degrees of belief means that the higher her degree of belief, on Monday, in the truth that it is Monday, the higher her degree of belief, on Tuesday, in the falsehood that it is Monday. In a certain sense, being closer to the truth on Monday brings the possible cost of being farther from the truth on Tuesday. This is an odd situation. Beauty is barred from the epistemically desirable goal of both believing to degree 1 that it is Monday on Monday, and believing to degree 0 that it is Monday on Tuesday. In this odd situation the best compromise, with respect to the claim that it is Monday, might well be to believe to degree 1 that it is Monday whenever she awakens. She will be surely be right on Monday, and possibly never be wrong about the day during the experiment. ${ }^{5}$

\footnotetext{
${ }^{2}$ Indeed this is how Elga[10] describes the memory erasure.

${ }^{3}$ If a yoked degree of belief changes while Beauty is awake on Monday, that degree of belief is to change similarly on Tuesday (if she is awakened on Tuesday).

${ }^{4}$ As the case is sometimes described, Beauty's experiences on Monday and Tuesday are phenomenally indistinguishable. If she sees a triangular shadow on the wall on Monday, she sees a triangular shadow on the wall on Tuesday. If she hears a passing airplane on Monday, she hears a passing airplane on Tuesday. And so on. As several have noted (e.g. Kierland and Monton[20], Stalnaker[29] and Titelbaum[30]), the puzzle remains even if her experiences are not phenomenally indistinguishable, as long as the difference between her experiences provide no evidence about propositions of interest - such as propositions about the coin toss or the current day. One can accommodate this observation by yoking together degrees of belief only for propositions of interest as opposed to all propositions.

${ }^{5}$ One might attempt to formalize and thereby clarify this thought that optimism is the best compromise by using a scoring rule. However, an initial attempt is inconclusive. According to some scoring rules (for example a linear rule), $P(M O N)=1$, but by other scoring rules (for example Brier's rule) $P(M O N) \neq 1$. It is unclear which rule to apply in this case, and moreover, it is unclear how to calculate the score for Beauty's unusual circumstance. Kierland and Monton[20] have approached the Sleeping Beauty problem by using a scoring rule. They do not reach a single conclusive answer. They use Brier's rule to measure the
} 
Although suggestive, these thoughts are obviously not sufficient to show that Beauty should adopt the optimistic policy. I will later present an argument that $P(M O N)=1$. At the moment, I merely conclude that the claim that $P(M O N)=1$ should not be immediately rejected. So let us accept $P(M O N)=1$ as temporary working hypothesis. Given that hypothesis, we next see what goes wrong with the best thirder arguments.

\section{Problems with two thirder arguments}

According to thirders, $P(H E A D S)=1 / 3$. Two of the best $\operatorname{arguments}^{6}$ rely on the key premise

(EQ) $P\left(H_{M O N}\right)=P\left(T_{M O N}\right)=P\left(T_{T U E}\right)$.

For from (EQ) and

$$
P\left(H_{M O N}\right)+P\left(T_{M O N}\right)+P\left(T_{T U E}\right)=1
$$

it follows that $P\left(H_{M O N}\right)=1 / 3$, and given that

$$
P(H E A D S)=P\left(H_{M O N}\right)
$$

it follows that $P(H E A D S)=1 / 3$, the thirder's conclusion.

Given the agreed assumptions (in Section 2), optimism is incompatible with the key premise $(\mathrm{EQ})$ : if $P(M O N)=1$, then $P\left(H_{M O N}\right) \neq P\left(T_{M O N}\right)$ or $P\left(T_{M O N}\right) \neq P\left(T_{T U E}\right)$ (or both).

But what, in the eyes of the optimist, is wrong with thirder arguments for (EQ)?

\subsection{Elga's defense of (EQ)}

Elga[10] defends $(\mathrm{EQ})$ by arguing both that $P\left(H_{M O N}\right)=P\left(T_{M O N}\right)$, and that $P\left(T_{M O N}\right)=$

$P\left(T_{T U E}\right)$. The optimist need have no quarrel with Elga's argument for the former: Sup-

expected inaccuracy of Beauty's degree of belief that the coin lands heads. They argue that if expected total inaccuracy is calculated the answer is $1 / 2$, and if expected average inaccuracy is used, the answer is $1 / 3$. They don't mention that other plausible scoring rules yield yet other answers.

${ }^{6}$ by Elga[10], and by Arntzenius[1][2] and Dorr[8] 
pose, after awakening on Monday, Beauty is told that it is Monday. At that point her degree of belief that the coin lands heads should be $1 / 2$, for her degree of belief that the coin lands heads should be the same as the chance of the future event that the coin lands heads. (This is another application of the Principal Principle.) ${ }^{7}$ But when she is told that it is Monday, Beauty should revise her degrees of belief by conditionalization. In particular, her new degree of belief that $H_{M O N}$ should be $P\left(H_{M O N} / M O N\right)$. So $P\left(H_{M O N} / M O N\right)=1 / 2$, and it follows that $P\left(H_{M O N}\right)=P\left(T_{M O N}\right)$.

The error - from the point of view of the optimist - lies in Elga's defense of the other claim, that $P\left(T_{M O N}\right)=P\left(T_{T U E}\right)$. Elga reasons like this: suppose when Beauty wakes up Monday that she is informed that the coin landed tails. She thus learns that either $T_{M O N}$ or $T_{T U E}$ is true. Since she has no reason to favor $T_{M O N}$ over $T_{T U E}$, or $T_{T U E}$ over $T_{M O N}$, she should divide her credence equally between $T_{T U E}$ and $T_{M O N}$. According to Elga, this is an application of a "highly restricted principle of indifference". Since Beauty should update her beliefs by conditionalization, after learning that the coin landed tails her credence in $T_{M O N}$ ought to be the same as the conditional credence $P\left(T_{M O N} \mid T_{M O N}\right.$ or $\left.T_{T U E}\right)$, and her credence in $T_{T U E}$ ought to be the same as the conditional credence $P\left(T_{T U E} \mid T_{M O N}\right.$ or $\left.T_{T U E}\right)$. It follows that $P\left(T_{M O N} \mid T_{M O N}\right.$ or $\left.T_{T U E}\right)=P\left(T_{T U E} \mid T_{M O N}\right.$ or $\left.T_{T U E}\right)$, and therefore $P\left(T_{M O N}\right)=$ $P\left(T_{T U E}\right)$.

The optimist does not accept this reasoning. One of Elga's claims is that Beauty should divide her credence equally between $T_{T U E}$ and $T_{M O N}$ after learning TAILS. If so, then Beauty's credence in $M O N$ after learning TAILS should be less than 1 . For if she should divide her credence equally between $T_{T U E}$ and $T_{M O N}$, then her credence in $T_{M O N}$ should be less than 1 , and, therefore, her credence in $M O N$ should be less than 1 .

\footnotetext{
${ }^{7}$ We assume that, whether the coin toss occurs on Monday evening or Sunday evening, there is no difference to Beauty's credences.
} 
However, Elga also claims that after learning TAILS her credence in $T_{M O N}$ ought to be the same as her conditional credence $P\left(T_{M O N} \mid T_{M O N}\right.$ or $\left.T_{T U E}\right)$. If so, and if Beauty should have degree of belief 1 that it is Monday when she awakens, then upon learning TAILS, Beauty's credence that it is Monday ought to remain 1.

So, on pain of accepting that on learning TAILS Beauty should both have degree of belief 1 that it is Monday, and have degree of belief lower than 1 that it is Monday, the advocate of optimism should not accept both of Elga's claims.

It is open to the optimist to reject Elga's view that after learning TAILS Beauty's credence in $T_{M O N}$ ought to be the same as her conditional credence $P\left(T_{M O N} \mid T_{M O N}\right.$ or $\left.T_{T U E}\right)$. But this would seem to be an ad hoc departure from standard conditionalization.

A better route for the optimist is to reject Elga's claim that Beauty should divide her credence equally between $T_{T U E}$ and $T_{M O N}$ after learning TAILS; the optimist should reject Elga's "highly restricted" indifference principle. ${ }^{8}$

\subsection{Arntzenius and Dorr's argument for (EQ)}

Arntzenius and Dorr argue for (EQ) in a different way. They consider a variation of the original scenario: if the coin lands heads, Beauty is awakened on Tuesday, but a few moments after the awakening Beauty is given an unusual experience which indicates to her that it is Tuesday and the coin landed heads. The argument goes that when Beauty

\footnotetext{
${ }^{8}$ More formally, the principle, developed and defended in [11], is that similar centered worlds deserve equal credence. A centered world is a possible world with a designated time and individual. Different centered worlds $\mathrm{X}$ and $\mathrm{Y}$ are similar just in case $\mathrm{X}$ and $\mathrm{Y}$ are associated with the same possible world, and the designated individual of $\mathrm{X}$ (at the designated time) is in a state subjectively indistinguishable from the designated individual of $\mathrm{Y}$ (at the designated time). The problem with Elga's defense of the principle, from the perspective of the optimist, is that Elga argues, in effect, that if one should have at least some credence both in $\mathrm{X}$ and in similar world $\mathrm{Y}$, then one should have equal credence in $\mathrm{X}$ and $\mathrm{Y}$. But to defend the principle it needs to be further shown that if one should have at least some credence in $\mathrm{X}$ then one should have at least some credence in any possible world similar to X. However, the optimist thinks that it is rational to have credence in $T_{M O N}$ but no credence in the similar $T_{T U E}$. The optimist would be in good company in rejecting the indifference principle: see [31] for detailed criticism of Elga's view; Stalnaker [p.c.] has different reasons to worry about the application of this principle.
} 
is awakened on Monday, she should initially divide her credence equally among four possibilities: $P^{\prime}\left(H_{M O N}\right)=P^{\prime}\left(T_{M O N}\right)=P^{\prime}\left(H_{T U E}\right)=P^{\prime}\left(T_{T U E}\right)$. After waiting and failing to have the unusual experience, she is able to rule out $H_{T U E}$. Since she should update her beliefs by conditionalization, her revised credences should be divided equally among the three remaining possibilities: $P^{\prime \prime}\left(H_{M O N}\right)=P^{\prime \prime}\left(T_{M O N}\right)=P^{\prime \prime}\left(T_{T U E}\right)$. Her situation now is relevantly like her situation in the original scenario, so (EQ) is true for the original scenario.

Again the optimist should not accept this reasoning. The optimist might hold that after waiting and failing to have the unusual experience, Beauty should not use conditionalization to update her beliefs. But this would seem to be an ad hoc departure from standard conditionalization.

A better route for the optimist is to deny that when Beauty fails to have the unusual experience she merely learns that $H_{T U E}$ is not true. The optimist may contend that she learns something else, something relevant to what degree of belief she should have that it is Monday. She learns that she is in a situation like the original scenario. But, says the optimist, in the original scenario she should have degree of belief 1 that it is Monday. Thus, when Beauty fails to have the unusual experience she learns MON. So, she should not revise her credences by conditionalizing on not- $H_{T U E}$. She should conditionalize on the strongest proposition learned, $M O N .^{9}$

\section{Another thirder argument}

Michael Titelbaum[30] presents a different argument for the thirder view. Titelbaum develops a formal framework for rational belief revision which, unlike the simple Bayesian rule of updating by conditionalization, can model cases when a degree of belief changes from an extreme value (0 or 1$)$ to a non-extreme value. However, we

\footnotetext{
${ }^{9}$ Another possibility for the optimist is (like Bradley[5]) to deny that her situation in the revised story is relevantly like her situation in the original story.
} 
need not examine the details to note that as Titelbaum tells the story, when Beauty awakens she is "uncertain whether it is Monday or Tuesday". And when he formalizes what he calls the "Extrasystematic constraints" of the story, one explicit constraint is that $0<P(M O N)<1$. Thus, Titelbaum simply assumes that $P(M O N) \neq 1 .{ }^{10}$

Perhaps Titelbaum - or any other thirder - would insist that the claim that $0<P(M O N)<1$ is simply a stipulation. Isn't one free to stipulate anything one likes?

In reply: One can certainly stipulate that Beauty has a non-extreme degree of belief that it is Monday when she wakes up on Monday. But that is not enough for the thirder's needs. The thirder needs to make the case that Beauty should have a non-extreme degree of belief that it is Monday.

To see why, compare the following case. Stipulate that on Sunday, Confused Cal has degree of belief $1 / 3$ that Cleveland won the pennant. Also stipulate that on Monday, Cal has degree of belief 1/2 that Cleveland won the pennant, and that between Sunday and Monday, Cal doesn't lose relevant evidence, doesn't gain relevant evidence, and his cognitive faculties remain in order.

Does the case of Cal show that INERTIA is false? Clearly not. Now revise the case by removing the phrase "doesn't gain relevant evidence" from the previous paragraph. Does the revised case show that, given INERTIA, Cal gains relevant evidence? Again, clearly not. Cal might gain no relevant evidence, and might have degree of belief $1 / 2$ on Monday as stipulated, but it might not be that he should have that degree of belief. In order to justifiably conclude that Cal gains relevant evidence, an argument is needed

\footnotetext{
${ }^{10}$ Curiously Titelbaum is unusual in making this assumption explicit. When the Sleeping Beauty puzzle was introduced to the philosophical literature by Elga[10], the case was described without any mention of Beauty's degrees of belief about the day when she wakes. Nor does Lewis[22], one of the earliest halfers, mention any such thing. Later, something crept into the presentation of the situation. Dorr[8] says that when she awakes she "will not be able to tell that it is not Monday" and then assumes without argument that $P(M O N) \neq 1$. Hitchcock[14] says that when she wakes up she "will not know whether it is Monday or Tuesday". But many others, when describing the case, do not explicitly mention anything about Beauty being uncertain about the day when she awakes.[1][13][15][25][26][32][34]
} 
that Cal should have degree of belief $1 / 2$ on Monday. Merely stipulating that he has degree of belief $1 / 2$ is not sufficient.

The lesson here applies to Beauty. Suppose one stipulates that she has degree of belief $<1$ that it is Monday when she wakes up on Monday. And, for the sake of argument, suppose that it then follows (given well accepted principles and the other stipulations about the case) that she has degree of belief $1 / 3$ on Monday that the coin lands heads. Should one conclude by INERTIA that Beauty gains relevant evidence? No. Beauty might gain no relevant evidence, and might have degree of belief $<1$ that it is Monday when she wakes up on Monday, but it might not be that she should have that degree of belief. In order to justifiably conclude that Beauty gains relevant evidence, an argument is needed that Beauty should have degree of belief $<1$ that it is Monday when she wakes up on Monday. Merely stipulating that she has degree of belief $<1$ is not sufficient.

\section{A Dutch book}

Let us consider one last thirder argument. Some thirders support their view using a diachronic Dutch book. Although the force of such arguments is debatable, ${ }^{11}$ it will be instructive to discuss a recent example offered by Draper and Pust[9]. Note that Draper and Pust discuss a slightly different version of the Sleeping Beauty case. This version is just like the original, except that on Monday afternoon Beauty is told that it is Monday. Let us suppose that this additional element does not affect what Beauty's degree of belief should be when she wakes up on Monday, or anything else important about the original case. Note also that they ensure that the bookie does not have any information that Beauty lacks. Otherwise the bookie might be able to use that information to his advantage in betting. So the bookie will undergo the same procedure

\footnotetext{
${ }^{11}$ See e.g. Christensen[7]
} 
as Beauty, sleeping and waking at the same time, as well as memory erasing Monday night, if necessary.

Here is a version of Draper and Pust's diachronic Dutch book argument: The bookie sells Beauty two bets. On Sunday the bookie sells her a bet for $\$ 15$ that pays $\$ 30$ if the coin lands tails. Beauty considers this fair because on Sunday she has degree of belief $1 / 2$ that the coin lands tails. Beauty and the bookie then go to sleep. On Monday afternoon, Beauty and the bookie are told that it is Monday. Beauty revises her belief state. At this point, the bookie sells Beauty a bet for $\$ 30 z$ that pays $\$ 30$ if the coin lands heads, where $z$ is Beauty's revised degree of belief that the coin lands heads. Beauty considers this fair too. If she accepts these bets, then, if $z>1 / 2$, Beauty is guaranteed to lose. Whether the coin lands heads or tails Beauty loses $\$ 15-\$ 30 z$. (If $z<1 / 2$ then a different pair of bets will do the trick: On Sunday the bookie sells her a bet for $\$ 15$ that pays $\$ 30$ if the coin lands heads. On Monday, after Beauty and the bookie are told that it is Monday, the bookie sells Beauty a bet for $\$ 30(1-z)$ that pays $\$ 30$ if the coin lands tails.) Thus, if $z \neq 1 / 2$, Beauty is vulnerable to a diachronic Dutch book. And, we can see, if $z=1 / 2$ then Beauty comes out even, and thus avoids this Dutch book. So, if Beauty ought not to be vulnerable to a Dutch book, then, after Beauty is told that it is Monday, she ought to have degree of belief $1 / 2$ that the coin lands heads.

A further step is needed to reach the thirder view: if $P(H E A D S)=1 / 3$ then the Dutch book is avoided, and if $P(H E A D S) \neq 1 / 3$ then Beauty is vulnerable to the Dutch book. This further step seems plausible, if we suppose that when Beauty learns that it is Monday, she updates her belief by conditionalization, that is, if we suppose that $z=P(H E A D S \mid M O N)$. The thirders we have discussed all hold that $P(H E A D S)=P\left(H_{M O N}\right)=P\left(T_{M O N}\right)=P\left(T_{T U E}\right)=1 / 3$. So $P(M O N)=$ $P\left(H_{M O N}\right)+P\left(T_{M O N}\right)=2 / 3$, and thus $P(H E A D S \mid M O N)=P\left(H_{M O N}\right) / P(M O N)=$ 
$(1 / 3) /(2 / 3)=1 / 2$. So these thirders avoid the Dutch book. ${ }^{12}$ The halfer, as defended by David Lewis[22], thinks that $P(H E A D S)=P\left(H_{M O N}\right)=1 / 2$, and $P\left(T_{M O N}\right)=$ $P\left(T_{T U E}\right)=1 / 4$. So, $P(M O N)=P\left(H_{M O N}\right)+P\left(T_{M O N}\right)=3 / 4$, and $P(H E A D S \mid M O N)=$ $P\left(H_{M O N}\right) / P(M O N)=(1 / 2) /(3 / 4)=2 / 3$. So the Lewisian halfer faces this Dutch

book.

However, it is not only a thirder who can avoid Draper and Pust's Dutch book. The optimist halfer avoids it too. According to the optimist halfer, $P(H E A D S)=1 / 2$ and $P(M O N)=1$. So $P(H E A D S \mid M O N)=1 / 2 .{ }^{13,14}$

\footnotetext{
${ }^{12}$ Notice that any thirder who accepts that $P(H E A D S \mid M O N)=1 / 2$ must hold that $P(M O N)<1$. For if $P(H E A D S \mid M O N)=1 / 2$ then $P\left(H_{M O N}\right)=P\left(T_{M O N}\right)$. So, since, $P(H E A D S)=P\left(H_{M O N}\right)=1 / 3$, $P(M O N)=2 / 3$.

${ }^{13}$ Hitchcock[14] presents a different Dutch book argument against the halfer view: On Sunday Beauty pays $\$ 15$ for a bet that yields $\$ 30$ if tails, and whenever she wakes up she pays $\$ 10$ for a bet that yields $\$ 20$ if heads. If the coin lands heads she loses $\$ 15$ on Sunday and gains $\$ 10$ on Monday for a net loss of $\$ 5$. If the coin lands tails she gains $\$ 15$ on Sunday and loses $\$ 10$ on both Monday and Tuesday for a net loss of $\$ 5$. This argument is criticized by both Bradley and Leitgeb[6] and by Draper and Pust. Draper and Pust rightly point out that (at least on evidential decision theory) the halfer should not consider the bets offered when she wakes to be fair. That is easy to see in the case of optimist halfer Beauty. Since optimist halfer Beauty is certain that it is Monday whenever she wakes, she is certain that if she were to accept the bet today she would accept it tomorrow (if awakened). So she is certain that if she accepts the bet then if the coin lands heads she wins $\$ 10$, and if the coin lands tails she loses $\$ 10$ both today and tomorrow. That means the expected value of the bet is $P(H E A D S) * \$ 10+P(T A I L S) *(-\$ 20)=.5 * \$ 10-.5 * \$ 20=-\$ 5$. Since the expected value is less than 0 the bet is not fair. Even some causal decision theorists may agree, because (1) Beauty's activities on Monday do in some way causally influence the state of the world on Tuesday and (2) When she wakes Beauty is certain that if she were to accept the bet today she would accept it tomorrow (if awakened).

${ }^{14}$ One might think that there is a simple diachronic Dutch book against the optimist halfer's view that $P(M O N)=1$ because with that degree of belief Beauty would bet at any odds that it is Monday. An audience member at a conference suggested the following. Since Beauty is certain that it is Monday whenever she wakes, on both Monday and Tuesday (if awakened) she should be willing to pay $\$ 30$ for a bet that yields $\$ 40$ if it is Monday. But then one can guarantee a sure loss by adding another bet on Sunday: pay $\$ 15$ for a bet that yields $\$ 30$ if the coin lands tails. Whether the coin lands heads or tails Beauty is sure to lose. If the coin lands heads she loses $\$ 15$ on the Sunday bet and gains $\$ 10$ on the Monday bet for a net loss of $\$ 5$. If the coin lands tails she gains $\$ 15$ on the Sunday bet and $\$ 10$ on the Monday bet, but loses $\$ 30$ on the Tuesday bet, for a net loss of $\$ 5$.

This Dutch book argument is problematic for reasons similar to those in the previous footnote. Beauty would not accept the bets on Monday and Tuesday as fair. Whenever she awakes, she is certain that it is Monday, so she is also certain that if she accepts the bet today she will gain $\$ 10$. However, she is also certain that, if the coin lands tails, if she accepts today she will accept the same bet tomorrow and lose $\$ 30$. So, since she has degree of belief .5 that the coin lands heads, the expected value of this bet is $.5 * \$ 10+.5 *(\$ 10-\$ 30)=-\$ 5$. The expected value of the bet is less than 0 , so it is not fair (at least according to evidential decision theory and possibly some versions of causal decision theory as well.)
} 


\section{An argument for optimism}

We have now seen the best available arguments for the thirder view, and where, in the optimist's view, these arguments go wrong. It is now time to argue in favor of optimism. Two premises entail that $P(M O N)=1$ : (1) $P(H E A D S / M O N)=1 / 2$; (2) $P(H E A D S)=1 / 2 .{ }^{15}$

We have just seen two explicit arguments for (1), the Dutch book argument in the previous section, and the following argument by Elga: after Beauty is told that it is Monday, her degree of belief that the coin lands heads should be $1 / 2$, for her degree of belief that the coin lands heads should be the same as the chance of the future event that the coin lands heads. But when she is told that it is Monday, Beauty should revise her degrees of belief by conditionalization. In particular, her new degree of belief that $H E A D S$ should be $P(H E A D S / M O N)$.

Now recall the apparently compelling halfer reasoning in favor of (2): Since Beauty knew that the coin was fair, on Sunday she should have degree of belief $1 / 2$ that the coin lands heads. When Beauty awakens on Monday she learns nothing relevant to the coin toss. She does learn that she is awake, but surely that news is not relevant to the coin toss. Moreover, she has no cognitive mishap between Sunday and Monday, and does not forget anything or lose relevant evidence in some other way. So, by INERTIA, when she wakes up she should continue to have degree of belief $1 / 2$ that the coin lands heads.

Optimists conclude from $(1)$ and $(2)$ that $P(M O N)=1$. There are strong arguments in favor of (1) and (2), and (1) and (2) together entail $P(M O N)=1$. Such is the compelling reasoning for the optimist view that $P(M O N)=1$.

Thirders disagree. Thirders accept $(1)$ but think $P(M O N)<1$. So thirders would

\footnotetext{
${ }^{15}$ It follows that $P(H E A D S)=P(H E A D S / M O N)=P(H E A D S \& M O N) / P(M O N)$. But $P(H E A D S \& M O N)=P(H E A D S)$. So $P(H E A D S)=P(H E A D S) / P(M O N)$, and thus $P(M O N)=1$. (This reasoning requires that $P(M O N)>0$, but this must be so since $P(H E A D S / M O N)=1 / 2$.)
} 
take the optimist argument as a reductio showing that (2) is false, and conclude therefore that Beauty gains or loses relevant evidence (or both) when she awakes, or else INERTIA is false.

Which reasoning should we accept, the optimist's or the thirder's? The optimist's reasoning relies on the premise that Beauty neither gains nor loses relevant evidence when she awakes. The conclusion of this reasoning, $P(M O N)=1$, is surprising. Thirders, as we have seen, hold that $P(M O N)<1$. Thirders conclude, surprisingly, that Beauty gains relevant evidence when she awakes, or loses relevant evidence, or, even more surprisingly, that INERTIA is false. In short: thirders claim that $P(M O N)<$ 1, and conclude that Beauty gains or loses relevant evidence (or conclude, less plausibly, that INERTIA is false); optimists claim that Beauty does not gain or lose relevant evidence, and conclude that $P(M O N)=1$. Thirders and optimists both start with an intuitive claim, and reach a counterintuitive conclusion. It is intuitive that $P(M O N)<$ 1. (Wouldn't Beauty wonder what day it is? Wouldn't she learn something if she is told that it is Monday?) However it is counterintuitive that on Monday she gains or loses evidence relevant to the coin flip. (How can what she learns on Monday be relevant to the coin flip? And how can she lose relevant evidence? She doesn't forget anything or have a cognitive mishap - how can she lose relevant evidence in some other way?) On the other side, it is intuitive that what she learns on Monday is not relevant to the coin flip and it is intuitive that she doesn't lose any evidence- much less lose evidence relevant to the coin flip. But it is counterintuitive that $P(M O N)=1$. As far as intuitions go, the optimist's reasoning and the thirder's reasoning are on a par, with intuitive premises and a counterintuitive conclusion. Moreover the optimist and the thirder accept the same principles: belief revision by simple conditionalization, the Principal Principle, and (at least most thirders anyway) InERTIA. Thus, as far as intuitions and principles go, the optimist argument is at least as persuasive as any thirder argument we have seen. In itself that is an interesting conclusion - until now 
the optimist view has been rejected by all without discussion.

Indeed, further consideration tips the balance in favor of the optimist's argument. Notwithstanding numerous thirder arguments, convincing to many, the Sleeping Beauty problem continues to rankle. Despite the thirder arguments, it is still difficult to believe that Beauty learns something relevant to the coin flip when she awakes on Monday. Beauty learns that she is awake, but she already knew on Sunday that she would learn that. What does she learn that is relevant to the coin flip? Moreover, it is difficult to believe that Beauty loses evidence relevant to the coin flip. She does not forgot anything between Sunday and Monday. She suffers no cognitive mishaps. What evidence does she lose and how does she lose it?

It does not look promising to claim that Beauty gains or loses relevant evidence. Even so, some thirders do claim so and attempt to explain how it is that Beauty gains or loses relevant evidence. Let us next explore the most serious of such attempts. They are, as we will see, unsuccessful.

\subsection{Does Beauty gain relevant evidence when she awakes?}

The most developed attempt to explain how Beauty learns something relevant to the coin flip is due to Horgan.[16][17] According to Horgan, Beauty learns "I was awakened today by the experimenters", and this information is relevant to the coin flip. Horgan tries to isolate the effect of what Beauty learns by partitioning the current information that she has on Monday after being awakened. When Beauty wakes up on Monday, she first assigns preliminary probabilities relative to a portion of her current information. This portion of her current information excludes the information that she was awakened today by the experimenters and also excludes the information that she is awake right now. The portion includes the information about the situation that Beauty had on Sunday and continues to have on Monday, and also includes the information that today is either Monday or Tuesday.[16, 53] According to Horgan, $H_{M O N}, T_{M O N}, H_{T U E}$ 
and $T_{T U E}$ are all consistent with and equally well supported by that portion of her current information. Since, he argues, these four possibilities are mutually exclusive and exhaustive, the preliminary probabilities should be: $P^{-}\left(H_{M O N}\right)=P^{-}\left(T_{M O N}\right)=$ $P^{-}\left(H_{T U E}\right)=P^{-}\left(T_{T U E}\right)=1 / 4$. And thus the preliminary probability of HEADS is $P^{-}\left(H_{M O N}\right)+P^{-}\left(H_{T U E}\right)=1 / 2$.

Next, according to Horgan, Beauty should update her preliminary probabilities by conditionalization on the information that she has been awakened today. That information (together with what she knows about the experiment) rules out $H_{T U E}$. Conditionalizing on this information yields: $P\left(H_{M O N}\right)=P\left(T_{M O N}\right)=P\left(T_{T U E}\right)=$ $1 / 3$, and so $P(H E A D S)=P\left(H_{M O N}\right)=1 / 3$. On this view, what Beauty learns when she wakes is relevant to the coin flip because it lowers the preliminary probability $1 / 2$ of $H E A D S$ to the updated probability $1 / 3$.

It is unclear to me whether this two-stage framework is a generalization of standard Bayesian conditionalization (as Horgan suggests) or is instead a departure from it. But let us accept the framework for the sake of argument. Even so, the proposal fails. A minor addition to the Sleeping Beauty story will help show why. Suppose that on Sunday a poster advertising a performance of the ballet Sleeping Beauty is hanging on the door outside Beauty's room in the experimental laboratory. The poster will remain on the door at least until Monday evening. The poster cannot be seen by Beauty from inside the room. If the coin lands tails, the poster remains on the door throughout the experiment. However, if the coin lands heads, the poster is removed on Monday evening at midnight while Beauty is sleeping, and then returned to its place twenty-four hours later, as Beauty continues to sleep. Beauty is certain of all of this. On Sunday, before she enters the room, she sees the poster on the door and she is certain that the poster is on the door. Throughout the experiment she will continue to be certain that the poster is on the door. Note that the addition of the poster does not matter for any 
of the thirder arguments discussed earlier. ${ }^{16}$

In Horgan's framework, Beauty's preliminary probabilities on Monday are determined relative to a portion of her current information which includes the information about the situation that Beauty had on Sunday and continues to have on Monday, and also includes the information that today is either Monday or Tuesday. Two pieces of information that Beauty has on Sunday and continues to have on Monday are: (a) the poster is on the door, and (b) on Tuesday, if the coin lands heads, the poster is not on the door. From this information, it follows that $H_{T U E}$ is ruled out, and therefore the preliminary probability of $H_{T U E}$ is 0 . That means that what Beauty learns when she wakes is not relevant to $H E A D S$; updating her preliminary probabilities by conditionalization on the information that she has been awakened today has no effect on the probability of $H_{M O N}$ and thus no effect on the probability of $H E A D S$.

Horgan may respond by excluding the information that the poster is on the door from the portion of her information used to calculate her preliminary probabilities. But that response does not work. ${ }^{17}$ A second response is to hold that while poster

\footnotetext{
${ }^{16}$ For example, if the arguments mentioned for the thirder premise that $P(H E A D S / M O N)=1 / 2$ work for the original story, they work for the poster version. Being certain that the poster is on the door doesn't change whether Beauty is subject to Draper and Pust's diachronic Dutch book. Being certain that the poster is on the door doesn't affect Elga's reasoning based on the claim that if, on Monday, Beauty learns that it is Monday, she ought to have degree of belief $1 / 2$ that the coin lands heads.

${ }^{17}$ To see why, consider the effort of Pust[27], who also argues against Horgan that Beauty's preliminary probability in $H_{T U E}$ is 0 . Pust relies on the claim that it is not possible for Beauty to be in an epistemic situation in which she lacks the information that she is presently conscious. Horgan's reply, in effect, is to exclude her information that she is presently conscious from the portion of her current information used to determine her preliminary probabilities. Horgan imagines Beauty saying to herself on Monday, "Statements $H_{M O N}, H_{T U E}, T_{M O N}$, and $T_{T U E}$ are all consistent with the conjunction of (1) the information I had on Sunday and with (2) the information that today is either Monday or Tuesday. In particular, statement $H_{T U E}$ is consistent with that specific information - notwithstanding the fact that if $H_{T U E}$ were true, then today I would not be conscious. So, relative to the information just mentioned, and only that information, the four statements each have the same degree of evidential support." [17] (The names of the possibilities are changed to match the present paper.) Horgan does not explicitly say why he excludes Beauty's information that she is presently conscious. Presumably he thinks that Beauty's (Monday) information that she is presently conscious is not information that she had on Sunday. Or perhaps he thinks that the information that Beauty is presently conscious should be excluded because that information (given the description of the case) entails that she has been awakened today, and the information that she has been awakened today is already excluded. Either way, Horgan has no such grounds to exclude the information that the poster is on the door. Beauty has and continues to have the information that the poster is on the door from Sunday to
} 
Beauty does not gain any relevant information, standard Beauty does. But that second response is inadequate too: it would leave a thirder about poster Beauty with no explanation why her degree of belief in $H E A D S$ should change, and it would leave a halfer about poster Beauty with the challenge of explaining why the addition of the poster should make a difference to Beauty's degree of belief in $H E A D S$, when that addition does not affect the force of the thirder arguments discussed earlier.

Horgan's proposal aside, the poster case highlights a challenge facing any attempt to show that when she learns that she is awake she gains information relevant to $H E A D S$. In the poster version Beauty is certain that the poster is on the door and she remains certain that the poster is on the door throughout the experiment. Similarly, it seems, in the standard version, not only is she certain, on Sunday, that "Today is Tuesday and the coin lands heads" is false, throughout the experiment she remains certain that this sentence is false. But on Monday, certainty that "Today is Tuesday and the coin lands heads" is false is sufficient to rule out $H_{T U E}$. So the information that she is awake is not needed to rule out $H_{T U E}$. But then how could learning that she is awake be in any way relevant to $H E A D S ?^{18}$

Monday, and that information does not entail that she has been awakened today- the poster is also on the door on Monday while she is sleeping before being awakened. In essence, the problem for Horgan's view is that on Sunday Beauty already had information which, on Monday, suffices to rule out $H_{T U E}$; on Monday she can rule out $H_{T U E}$ without using the information that she has been awakened today.

Someone might suggest that the information conveyed on Sunday using "The poster is on the door" differs from the information conveyed on Monday using "The poster is on the door": the former describes the location of the poster on Sunday and the latter describes the location of the poster on Monday. But this suggestion does not help Horgan. Beauty has the information that the poster is on the door throughout the experiment, even if the nature of this information changes over time. We can put it this way: throughout the experiment she will remain certain that the sentence "The poster is on the door" is true even if the information conveyed by a use of this sentence changes over time. Note that she may need to be awake in order to fix the reference of the indexical "today" or the present tense "The poster is on the door" and thus she may need to be awake on Monday in order to eliminate $H_{T U E}$ on Monday. But that does not mean that she needs to use the information that she has been awakened (or that she is awake) to eliminate $H_{T U E}$.

${ }^{18}$ She may need to be awake in order to fix the reference of an indexical like "today" in "Today is Tuesday and the coin lands heads", but that does not mean that she needs to use the information that she is awake in order to fix the reference of "today" or to eliminate $H_{T U E}$. 


\subsection{Does Beauty lose relevant evidence when she awakes?}

Thus, the best developed attempt to show that Beauty gains relevant evidence does not succeed. The thirder might instead pursue another option: Beauty loses relevant evidence. ${ }^{19}$ This approach is not promising either. Between Sunday and Monday, Beauty does not forget anything, and she suffers no cognitive mishaps. Unless there is some other way to lose evidence, Beauty does not lose any.

Perhaps there is another way to lose evidence. The Shangri-La example (due to Arntzenius[1]) has been thought by some to show how:

You will travel to Shangri-La by one of two paths, the Path by the Mountain or the Path by the Sea. Which path you take is determined by the flip of a fair coin. If the coin lands heads you travel on the Path by the Mountain; if the coin lands tails you travel on the Path by the Sea. However, the guardians of Shangri-La have arranged it so that if the coin lands tails and you travel via the sea, you don't remember doing so: when you pass through the gates of Shangri-La, your memories of traveling by the sea are wiped out, and replaced by apparent memories of having traveled through the mountains. On the other hand, if the coin lands heads and you go via the mountains, nothing untoward happens and you enter the city with your memories intact. Before you set off, all the arrangements are known with certainty by you.

Before the coin is flipped, according to the Principal Principle, you should have degree of belief $1 / 2$ that the coin lands heads. Now suppose the coin lands heads and you travel by the mountains. As you are traveling, we can agree, you should be certain that the coin landed heads because you see the enjoyable mountain scene. Then, according to Arntzenius, after entering Shangri-La your degree of belief that the coin landed heads should be $1 / 2$ again: “... once you have arrived, you will revert to having degree of belief $1 / 2$ in heads. For you will know that you would have had the memories that you have either way, and hence you know that the only relevant

\footnotetext{
${ }^{19}$ The two options do not exclude each other; the thirder might claim that Beauty both gains and loses relevant evidence. But if Beauty does not gain relevant evidence she does not both gain and lose relevant evidence.
} 
information that you have is that the coin was fair." [1][p 356 $]^{20}$

If this is right, then if the coin lands heads you might be said to lose information upon passing through the gates of the city. Just before the gates you are certain that the coin landed heads and you are certain that you traveled through the mountains. After passing through the gates you are no longer certain of these things. This loss of certainty, one might say, is (or indicates) a loss of information. But you do not forget anything and you do not suffer any cognitive mishaps. Apparently then there is different a way to lose information- a way different from forgetting or suffering a cognitive mishap. The mere threat of being in the counterfactual situation where the coin lands tails and forgetting/disruption occurs seems to be enough to bring about this loss. Perhaps Beauty loses information in some such way; perhaps the threat of the possible memory erasure Monday evening is enough to bring about a loss of information about what day it is.

However, the Shangri-La case does not show how to lose information in a way different from forgetting or cognitive mishap. Arntzenius claims that, if the coin lands heads, after passing through the gate you should change from degree of belief 1 to degree of belief $1 / 2$ that the coin landed heads. But this claim is incompatible with the Bayesian principle that belief updating (for non-indexical propositions) should only occur by conditionalization. Given that principle, after passing through the gate you should continue to have credence 1 that the coin landed heads. For once you have credence 1 that the coin landed heads, your credence cannot be lowered by conditionalization. Indeed, intuitively, the principle yields the correct result in this case. Why should you reduce your credence in the truth that the coin landed heads because an unfortunate event would have occurred if the coin had instead landed tails?

On the other hand, there is some intuitive appeal to the view that your degree of

\footnotetext{
${ }^{20}$ Although Arntzenius says you will revert to degree of belief $1 / 2$, I take it that he also holds that you should revert to degree of belief $1 / 2$.
} 
belief should revert to $1 / 2$ after passing through the gates. (Imagine yourself in that situation. Wouldn’t you doubt whether your memories are accurate?) But principled commitment to Bayesian principles should not stand or fall on the basis of mere intuitive appeal - especially given the countervailing intuition that you should not give up your certainty in the truth that the coin landed heads because something untoward would have happened to you if the things had been different.

Arntzenius is of course aware that his view of the Shangri-La case conflicts with standard Bayesian principles. However mere intuition is not the justification he gives for his view. There you are before the gates. The coin landed heads and you are certain that it did. Then you pass through the gates. Arntzenius's suggestion seems to be that you should now ignore your evidence that the coin landed heads because you know that you would have had the same evidence whether the coin landed heads or the coin landed tails. ${ }^{21}$ But this way of reasoning is suspicious at best. Compare the following. You are, I take it, certain that you are awake right now. But suppose you start thinking about what it is like when you are dreaming and you realize that you would have had the same evidence whether you are awake or you are asleep having a particularly vivid dream. At that point, should you then ignore your evidence that you are awake? No. Surely it would be a mistake at that point to ignore your evidence that you are awake. For you are certain that you are not asleep; you are certain that you are not in the counterfactual situation where you are asleep having a particularly vivid dream. Learning the interesting fact that you would have had the same evidence in a certain counterfactual situation should not bring you to stop being certain that that situation is counterfactual. ${ }^{22}$

\footnotetext{
${ }^{21}$ This is the best sense I can make of the remark quoted earlier: " ... you will know that you would have had the memories that you have either way, and hence you know that the only relevant information that you have is that the coin was fair."

${ }^{22}$ The point here is simply that you should not, on the basis of discovering that you would have had the same evidence if you were dreaming, accept the conclusion that you should ignore your evidence that you are awake. We can save the problem of explaining what exactly is wrong with this reasoning for later. Here is another example of similar suspicious reasoning: The wall is painted red, and on Monday morning you
} 
One can say something similar about Shangri-La. At the gates of Shangri-La, after the coin landed heads, you are certain that the coin landed heads, and you are certain that you are not in the counterfactual situation where the coin landed tails. Then you pass through the gates. On the other side of the gates, you learn an interesting fact about the counterfactual situation in which the coin landed tails. You learn that the evidence you have at that point is the same (or at least appears the same) as the evidence you would have had in the counterfactual situation in which the coin landed tails. You should not then ignore your evidence that the coin landed heads. For you are certain that you are not in the counterfactual situation in which coin landed tails. You have merely learned an interesting fact about that counterfactual situation. ${ }^{23}$

Neither mere intuition nor Arntzenius' suspicious reasoning should sway us from principle: when the coin lands heads, you should continue to be certain that the coin landed heads after passing through the gates of Shangri-La. ${ }^{24}$ Thus the Shangri-La case does not provide good reason to think there is a way to lose evidence different from forgetting or suffering a cognitive mishap. So that case provides no good reason to think that Beauty loses evidence.

are certain that it is painted red. As you know, in a nearby room there is a white wall with a red light shining on it, but you are certain that you are not in that nearby room. It would be a mistake to ignore your evidence that the wall is painted red, even if you come to know on Monday evening that you would have had the same evidence whether you are in the red painted room or the red lighted room. For you are certain that you are not in the nearby room. Your certainty should not be shaken by the discovery of the interesting fact that you would have had the same evidence in some counterfactual situation.

${ }^{23}$ Perhaps behind Arntzenius's reasoning is a principle like this: if you have the same evidence that $\mathrm{p}$ in situation S as you have in situation S', then you should have the same degree of belief that $\mathrm{p}$ in situation $\mathrm{S}$ and situation S'. Even if this principle is correct, it is not sufficient to show that you should have degree of belief $1 / 2$ that the coin lands heads after passing through the gates. It is compatible with this principle that you have degree of belief 1 that the coin lands heads after passing through the gates whether the coin lands heads or tails.

${ }^{24}$ As far as I know this view of the Shangri-La case has not yet found a defender in print - although Wolfgang Schwarz apparently advocated it in a talk. 


\subsection{On the optimist side}

Earlier, we saw that as far as intuition and principles go, the reasoning of optimists and the reasoning of thirders are on a par. Thirders and optimists both start with an intuitive claim, and reach a counterintuitive conclusion. Thirders claim that $P(M O N)<1$, and conclude that Beauty gains or loses relevant evidence (or conclude, less plausibly, that INERTIA is false); optimists claim that Beauty does not gain or lose relevant evidence, and conclude that $P(M O N)=1$.

The last section investigated the most plausible attempt to show how Beauty can lose relevant evidence. The section before last examined the most developed attempt to show that Beauty gains relevant evidence. We found no good reason to believe that Beauty either gains or loses relevant evidence. This strongly supports the optimist's claim that Beauty does not gain or lose relevant evidence, given the lack of any better proposals to explain how Beauty can gain or lose relevant evidence. These considerations thus tip the balance in favor of the optimist's argument over the thirder's. One should follow the argument where it leads, and conclude that $P(M O N)=1$.

However, there is a lingering issue. The optimist's conclusion, $P(M O N)=1$, is not easy to believe. When one imagines oneself in Beauty's position upon awakening on Monday, one imagines that one would feel uncertain about what day it is. It is easy to think that Beauty should be uncertain about what day it is when she awakes on Monday.

However, the optimist can help make the claim that $P(M O N)=1$ easier to accept. The optimist can explain why one might mistakenly believe that $P(M O N)<1$. The explanation is that one is attracted to something like the following thought:

Because of the memory erasure, Beauty cannot distinguish Monday wakings from Tuesday wakings. When she awakes on Monday, she has no evidence that indicates to her that it is Monday as opposed to Tuesday. So, on Monday, surely she should give at least some credence to the proposition that it is now Tuesday. 
It is understandable that this sort of thought might lead one to believe that $P(M O N)<$

1. But, again, there is a striking similarity to a skeptical thought:

I cannot distinguish an experience had while awake from particularly vivid dream experiences had while asleep; I have no evidence that indicates to me that I am awake now as opposed to being asleep having one of those particularly vivid dreams. So surely I should give at least some credence to the proposition that I am asleep right now.

It is understandable that this sort of thought might lead me to uncertainty whether I am awake now. Many have been attracted to skeptical thoughts. However, while attractive, the skeptical thought is mistaken. I am certain that I am awake right now, and surely it is rational for me to be certain that I am awake right now. Despite recognizing that the thought is mistaken (we can figure out why another day) the skeptical thought retains an illusory air of cogency.

Similarly, says the optimist, the thought about Beauty is mistaken. On Monday, Beauty should be certain that it is Monday. But, as in the skeptical case, even after recognizing that the thought is mistake, the illusory air of cogency persists. ${ }^{25}$

\section{Conclusion}

I have considered the best thirder arguments I am aware of: by Elga, by Arntzenius and Dorr, by Titelbaum, and by Draper and Pust. I have explained how these arguments each require that $P(M O N)<1$. Therefore, if $P(M O N)=1$, these arguments should

\footnotetext{
${ }^{25}$ Is there is a quick way to show that although the skeptical thought is mistaken the thought about Beauty is not mistaken? One might say that although I have overwhelming evidence that I am awake now, Beauty (on Monday) does not have overwhelming evidence that it is Monday. But this suggestion fails. On Monday, Beauty does have overwhelming evidence that it is Monday- she is filled with memories of the happenings on Sunday, memories, as she would put it, about "yesterday". One might reply that she does not have overwhelming evidence that it is Monday because (1) she should doubt that her memory is reliable because if it is Tuesday she would have indistinguishable (apparent) memories, and (2) doubt about the reliability of her memory undermines or defeats her evidence that today is Monday. But then parallel points would seem to apply to the dreaming case: I do not have overwhelming evidence that I am awake because (1) I should doubt that my experiences reliably indicate that I am awake because if I am asleep I would have indistinguishable experiences, and (2) doubt about the reliability of my experience undermines or defeats my evidence that I am awake.
} 
not be accepted. I have also argued that $P(M O N)=1$. Thus, the thirder arguments should not be accepted. The path is clear to be a halfer.

\section{References}

[1] F. Arntzenius. Reflections on sleeping beauty. Analysis, 62(273):53-62, 2002.

[2] F. Arntzenius. Some problems for conditionalization and reflection. Journal of Philosophy, C(7):356-70, 2003.

[3] N. Bostrom. Sleeping beauty and self-location: A hybrid model. Synthese, 157(1):59-78, 2007.

[4] L. Bovens. Judy Benjamin is a Sleeping Beauty. Analysis, 70(1):23-26, 2010.

[5] D. Bradley. Sleeping beauty: a note on Dorr's argument for $1 / 3$. Analysis, 63(279):266-268, 2003.

[6] D. Bradley and H. Leitgeb. When betting odds and credences come apart: more worries for Dutch book arguments. Analysis, 66(290):119-127, 2006.

[7] D. Christensen. Clever bookies and coherent beliefs. Philosophical Review, 100(2):229-247, 1991.

[8] C. Dorr. Sleeping beauty: in defence of Elga. Analysis, 62(276):292-296, 2002.

[9] K. Draper and J. Pust. Diachronic dutch books and sleeping beauty. Synthese, pages $1-7,2008$.

[10] A. Elga. Self-locating belief and the sleeping beauty problem. Analysis, 60(266):143-147, 2000.

[11] A. Elga. Defeating Dr. Evil with self-locating belief. Philosophy and Phenomenological Research, 69(2):383-396, 2004.

[12] Don Fallis. Attitudes toward epistemic risk and the value of experiments. Studia Logica, 86(2):215-246, 2007.

[13] J. Halpern. Sleeping beauty reconsidered: Conditioning and reflection in asynchronous systems. Oxford Studies in Epistemology, 2006.

[14] C. Hitchcock. Beauty and the bets. Synthese, 139(3):405-420, 2004.

[15] T. Horgan. Sleeping beauty awakened: new odds at the dawn of the new day. Analysis, 64(281):10-21, 2004.

[16] T. Horgan. Synchronic Bayesian updating and the generalized sleeping beauty problem. Analysis, 67(293):50-59, 2007.

[17] T. Horgan. Synchronic Bayesian updating and the Sleeping Beauty problem: reply to Pust. Synthese, 160(1):155-159, 2008.

[18] C. Jenkins. Sleeping beauty: A wake-up call. Philosophia Mathematica, 13(2):194$201,2005$. 
[19] K. Karlander and L. Spectre. Sleeping Beauty meets Monday. Synthese, 2009.

[20] B. Kierland and B. Monton. Minimizing inaccuracy for self-locating beliefs. Philosophy and Phenomenological Research, 70(2):384-395, 2005.

[21] N. Kim. Sleeping Beauty and shifted Jeffrey conditionalization. Synthese, 168(2):295-312, 2009.

[22] D. Lewis. Sleeping beauty: reply to Elga. Analysis, 61(271):171-176, 2001.

[23] P. J. Lewis. Quantum sleeping beauty. Analysis, 67(293):59-65, 2007.

[24] P. J. Lewis. Credence and self-location. Synthese, 2009.

[25] C. J. G. Meacham. Sleeping beauty and the dynamics of de se beliefs. Philosophical Studies, 138(2):245-269, 2008.

[26] B. Monton. Sleeping beauty and the forgetful Bayesian. Analysis, 62(273):47-53, 2002 .

[27] J. Pust. Horgan on sleeping beauty. Synthese, 160(1):97-101, 2008.

[28] R. Selten. Axiomatic characterization of the quadratic scoring rule. Experimental Economics, 1(1):43-62, 1998.

[29] Robert Stalnaker. Our Knowledge of the Internal World. Oxford University Press, 2008 .

[30] Michael Titelbaum. The relevance of self-locating beliefs. Philosophical Review, 2008.

[31] B. Weatherson. Should we respond to evil with indifference? Philosophy and Phenomenological Research, 70(3):613-635, 2005.

[32] R. Weintraub. Sleeping beauty: a simple solution. Analysis, 64(281):8-10, 2004.

[33] J. Weisberg. Conditionalization, reflection, and self-knowledge. Philosophical Studies, 135(2):179-197, 2007.

[34] R. White. The generalized sleeping beauty problem: a challenge for thirders. Analysis, 66(290):114-119, 2006. 